\title{
Microstructures of a Hydrogen-Storage Ti-V-Cr Alloy Studied by STEM-EELS
}

\author{
I. Park, ${ }^{*}$ N. Terashita**, and E. Abe* \\ * Department of Materials Engineering, University of Tokyo, 7-3-1 Hongo, Bunkyo-ku, Tokyo 113 - \\ 8656, Japan \\ ** Japan Metals \& Chemicals Co., Ltd., 232 Ogun-machi, Nishiokitama-gun, Yamagata, 999-1351, \\ Japan
}

Hydrogen energy is one of the prominent candidates for future energy resource. $\mathrm{LaNi}_{5}$-based alloys and their derivatives have been considered as one of the best hydrogen storage materials. However, their capacity is still below 2 mass \%, which is not sufficient for the use of fuel cell vehicles [1]. Recently, it is found that $b c c$-based solid solution alloys reveal a significant potential to replace $\mathrm{AB}_{5}$ alloys. Vanadium-containing $b c c$ alloys can be activated under ambient conditions and show an excellent capacity of approximately 3 mass\% [2-6]. One of the major problems of these $b c c$-alloys is that the amount of retained hydrogen gradually increases with accumulating a number of chargedischarge cycles [7]. In our study, a $\mathrm{Ti}_{25} \mathrm{~V}_{20} \mathrm{Cr}_{50} \mathrm{Mo}_{5}$ bcc solid-solution alloy has been studied. Pressure-composition isotherms of the sample were measured from 1 st to 20th cycles. The maximum hydrogen capacity at the 1 st cycle was about $1.71 \mathrm{H} / \mathrm{M}$, but it is found that there still remains the hydrogen of $0.48 \mathrm{H} / \mathrm{M}$ even after the desorption process. For tracing the residual hydrogen sites, microstructures of the alloy were investigated using advanced scanning transmission electron microscopy (STEM) combined with electron energy loss spectroscopy (EELS). Figure 1(a) shows electron diffraction pattern of the alloy after the hydrogen charge-discharge cycles for 20 times. It is found that the diffraction spots were streaked along [100] direction, and they headed toward only the inside direction. We can presume that the residual hydrogen in the matrix form the local hydrides. Figure 1(b) shows annular bright-field (ABF) STEM image of the same sample. It is observed that the lattice reveals some mosaics, accompanying a large number of dislocations that may be induced to accommodate the local lattice strains. In the fast Fourier transformation (FFT) pattern obtained from the ABF image, some extra diffuse peaks indicated by red arrows were reproduced in addition to the peaks from the $b c c$ structure. They correspond to the diffused peaks in the electron diffraction pattern. Figure 2 exhibits the result of local chemical analysis by STEM-EELS. We further find that an extremely fine phase separation occurs, forming Ti-rich and Cr-rich regions at several unit-cell scales. It is presumed that the retained hydrogen atoms are trapped preferentially at the Ti-rich regions.

\section{References}

[1] Schalpbach, L. et al., Nature 414 (2001) 353.

[2] Maeland, A. J. et al., J. Phys. Chem. 68 (1964) 2197.

[3] J. J. Reilly et al., Inorg. Chem. 9 (1970) 1678.

[4] S. Ono et al., J. Less Common Metals 72 (1980) 159.

[5] J H. Iba et al., J. Alloys Comp. 21-24 (1997) 253.

[6] S.-W. Cho et al., J. Alloys Comp. 288 (1999) 294.

[7] Y. Nakamura et al., J. Alloys Comp. 311 (2000) 317.

[8] This research was supported by the Ministry of Education, Culture, Sports, Science and Technology, Japan. 

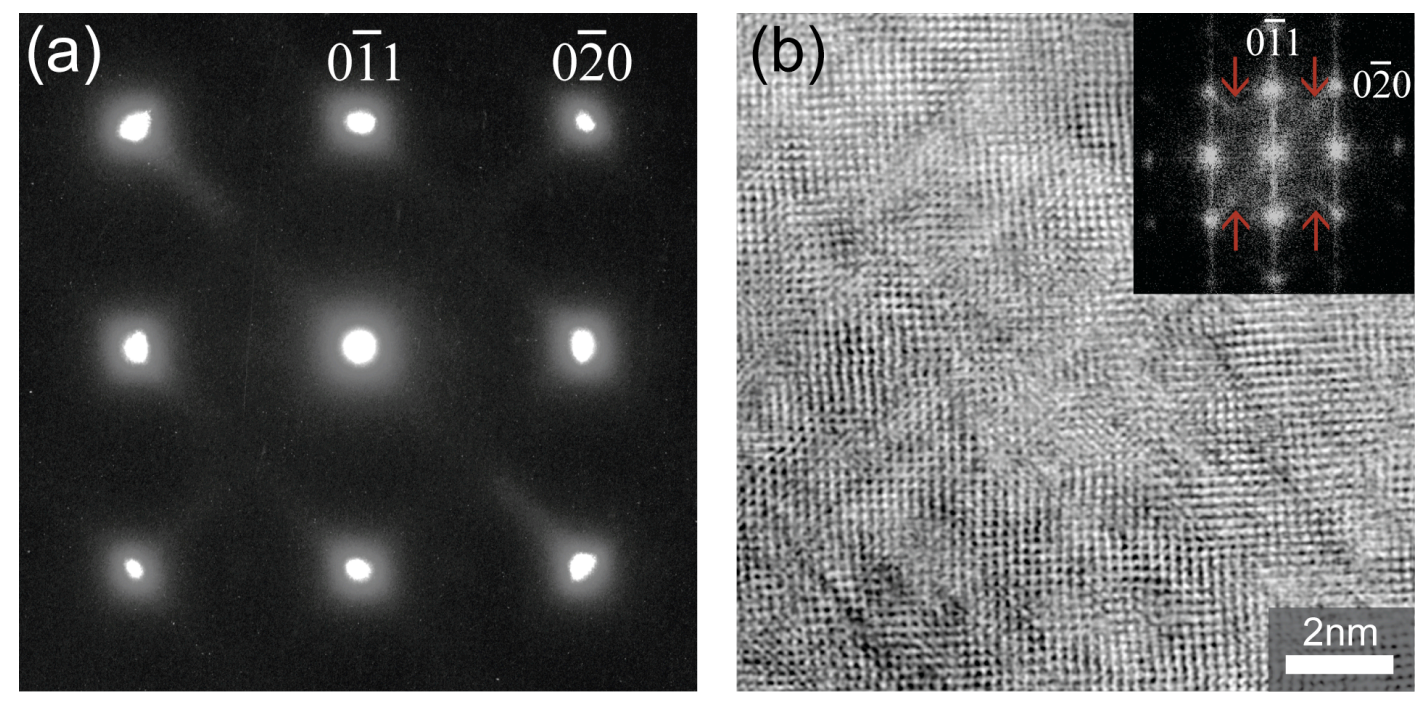

FIG. 1. (a) Electron diffraction pattern and (b) ABF-STEM image of the $\mathrm{Ti}_{25} \mathrm{~V}_{20} \mathrm{Cr}_{50} \mathrm{Mo}_{5} b c c$-alloy, which has been processed by the hydrogen charge-discharge cycles for 20 times.

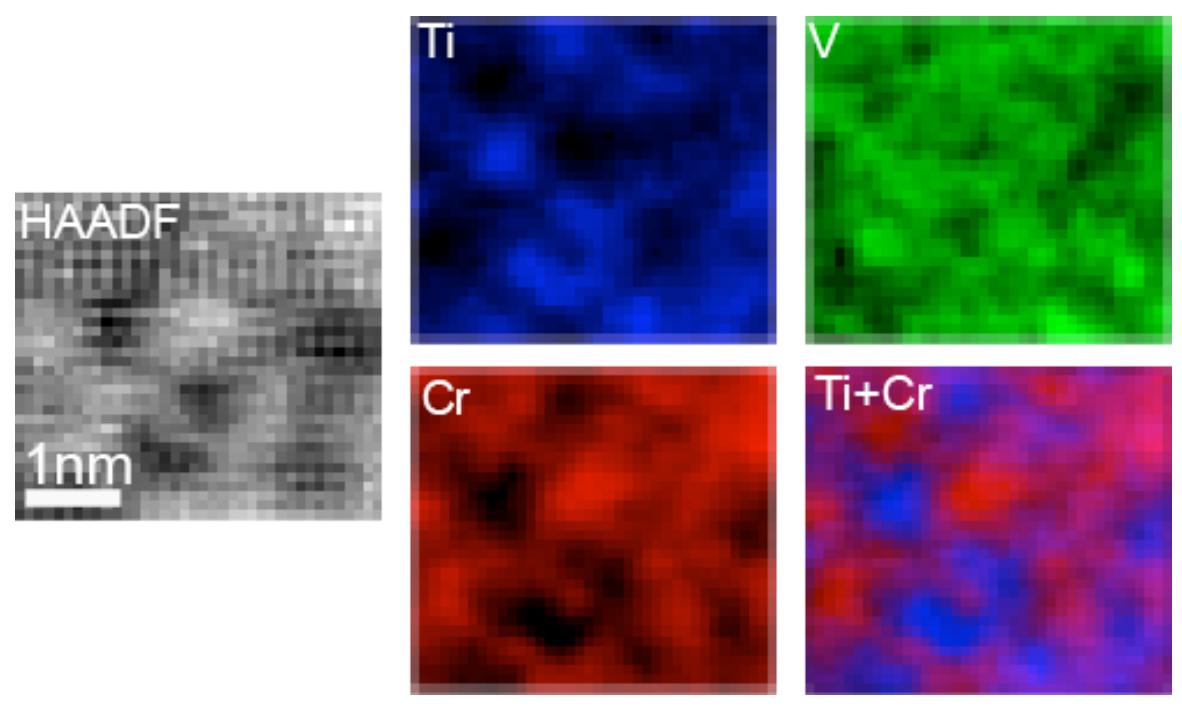

FIG. 2. STEM-HAADF and STEM-EELS images obtained simultaneously from the $\mathrm{Ti}_{25} \mathrm{~V}_{20} \mathrm{Cr}_{50} \mathrm{Mo}_{5}$ $b c c$-alloy, which has been processed by the hydrogen charge-discharge cycles for 20 times. 